\title{
Introduction to the Science Gateways and Portals Minitrack
}

\author{
Sandra Gesing \\ University of Notre Dame \\ Notre Dame, USA \\ sandra.gesing@nd.edu
}

\author{
Nancy Wilkins-Diehr \\ University of California \\ San Diego, USA \\ wilkinsn@sdsc.edu
}

\author{
Michelle Barker \\ Nectar \\ Parkville, Australia \\ Michelle.barker@,nectar.org.au
}

\begin{abstract}
Science gateways are online community spaces for science and engineering research, for humanities and education - mostly web-based resources for accessing data, software, computing services, and equipment specific to the needs of a science, engineering or humanities discipline. Creators of science gateways face a breadth of topics and manifold challenges, which necessitate close collaboration with the domain specialists but also calling in experts for diverse aspects. The Science Gateways and Portals minitrack brings together creators and domain specialists and provides the venue to present and discuss cutting-edge approaches, the challenges in this area and success stories.
\end{abstract}

\section{Introduction}

Science gateways are a community-specific set of tools, applications, and data collections that are integrated together via a web portal or a desktop application, providing access to resources, instruments and services, i.e., for distributed data management and distributed computing infrastructures. They offer easy and intuitive access to computing infrastructures and instruments irrespective of their location. The challenges in the area of gateways are manifold: from intuitive user interfaces and security features through efficient data and workflow management to parallelization of applications employing parallel and distributed architectures. In the last decade, quite a few innovations and developments have taken place on user interface layer and services layer and are reflected on infrastructure level in projects like XSEDE, NeCTAR, and local infrastructures. Diverse mature technologies are available and under active development: from portal frameworks through workflow-enabled grid/cloud portals and workbenches to platforms to ease the development of gateways supporting distributed computing infrastructures and access to data sources. Each approach has its own community and the implementations are designed to meet the specific needs and provide methodologies for analyzing data. The efficient support of communities demands to maintain and further develop the different approaches without re-inventing the wheel. Thus, it is crucial to bring together users, gateway developers and gateway providers to discuss problems and solutions in the area, to present lessons learned, to identify new issues, to shape future directions for research, foster the exchange of ideas, standards and common requirements and push towards the wider adoption of gateways.

The minitrack addresses the following topics in the presentations and the panel discussion

- Gateway enabling technologies and development frameworks

- $\quad$ Ready to use gateways in different areas and disciplines

- Management of high-throughput data via gateways

- Portal technology and portal construction methods

- Usage models and gateway tools in different disciplines

- $\quad$ Security aspects of gateways

- Usability studies of gateways

- Workflows and service composition in gateways

- Lessons learned

- Success stories

- The Science Gateways Community Institute serving scientists, researchers and developers 